\title{
ON THE DISTRIBUTION OF $\alpha p^{k}$ MODULO 1
}

\author{
by $\mathrm{K}$. C. WONG
}

(Received 31 Aug, 1995)

1. Introduction. The fractional part of the sequence $\left\{\alpha n^{k}\right\}$, where $\alpha$ is an irrational real number and $k$ is an integer, was first studied early this century, initiated by the work of Hardy, Littlewood and Weyl. It seems very natural to consider the subsequence $\left\{\alpha p^{k}\right\}$, where $p$ denotes a prime variable. The pioneering work in this direction was conducted by Vinogradov [13, 14]. Improvements have since been made by Vaughan [12], Ghosh [4], Harman $[6,7,8]$ and Jia [11]. The best results to date have been obtained by Harman for $k=1$ [9], by Baker and Harman for $2 \leq k \leq 12$ [1], and by Harman for larger $k$ [8]. In the following work, we shall adopt a sieve technique developed by Harman in [6] to show the following.

THEOREM. Let $\alpha$ be an irrational real number and write $\|x\|=\min _{n \in \mathbb{N}}|x-n|$. Then, for every real $\beta$, and every $\varepsilon>0$, there are infinitely many primes $p$ such that:

where

$$
\left\|\alpha p^{k}+\beta\right\|<p^{-\rho+\varepsilon}
$$

$$
\rho=\rho(k)= \begin{cases}5 / 56, & \text { if } k=3, \\ 1 / 21, & \text { if } k=4, \\ (0 \cdot 815) 2^{-k}, & \text { if } k \geq 5 .\end{cases}
$$

The above result is an improvement for $3 \leq k \leq 12$ of that of Baker and Harman in [1], who obtained $(2 / 3) 2^{-k}$. The constant 0.815 comes from a calculation which will be explained later in Section 4.

2. Notations and outline of method. Let $\varepsilon$ be a small positive constant and $\eta$ be another small positive constant, which is small compared to $\varepsilon$. The letter $p$ will always denote a prime. The following notations will be used:

$$
L=2 N^{\rho-\varepsilon / 2}, \delta=N^{-\rho+\varepsilon} / 2, \sigma=2^{k} \rho .
$$

Let $a / q$ be a convergent to the continued fraction expansion of $\alpha$ such that $\left(L N^{k}\right)^{1 / 2} \leq$ $q \leq\left(L(N+1)^{k}\right)^{1 / 2}$. Following [1], it suffices to prove that $\left\|a p^{k} / q+\beta\right\|<\delta$ with $p \leq N$. Write:

$$
\begin{aligned}
& B=\{n \in \mathbb{N}: n \leq N\}: \quad A=\left\{n \in B:(n, q)=1,\left\|a n^{k} / q+\beta\right\|<\delta\right\}, \\
& V(z)=\prod_{p<z}(1-1 / p), \quad P(z)=\prod_{p<z} p, \quad V_{1}(z)=\prod_{p<z}^{\prime}(1-1 / p),
\end{aligned}
$$

where $\Pi^{\prime}$ (or $\Sigma^{\prime}$ ) here and hereafter means that the product (or sum) is taken over primes that are coprime to $q$. For a subset $E$ in $\mathbb{N}$, the sieve function $S(E, z)$ and $E_{d}$ are defined as:

$$
\begin{gathered}
E_{d}=\{n: d n \in E\} ; \\
S(E, z)=|\{n \in E:(n, P(z))=1\}| ;
\end{gathered}
$$

Glasgow Math. J. 39 (1997) 121-130. 
« and $\gg$ are the usual Vinogradov's symbols and $m \sim M$ will mean $M \leq m<2 M$. As usual, write $\mathrm{e}(x)=\mathrm{e}^{2 i \pi x}$. We shall, using estimates for certain exponential sums, establish asymptotic formulae of the form:

$$
\sum_{m \sim M} a_{m} S\left(A_{m}, z\right)=2 \delta \sum_{m \sim M} a_{m} S\left(B_{m}, z\right)(1+R)
$$

where $R$ is an error of suitable size, for various ranges of $M$ and $z$. Using Buchstab's identity, we may decompose the sum $S\left(A, N^{1 / 2}\right)$ into single sums and double sums. It will turn out that we may give asymptotic formulae for each of the single sums and certain subsums of the double sums. The remaining double sums, being nonnegative, are discarded. Provided we have not discarded too much, this gives a positive lower bound for $S\left(A, N^{1 / 2}\right)$. This establishes the theorem.

3. Lemmas. In this section, we shall establish some lemmas which are essential in proving the theorem.

LEMMA 1. Let $\left\{a_{m}\right\}$ be a sequence of real numbers for $m \in B$, all of the same sign. Then

$$
\sum_{m \in A} a_{m}=2 \delta \sum_{m \in B} a_{m}+O\left(\delta \sum_{n \leq L} \mid \sum_{m \in B} a_{m} \mathrm{e}\left(\frac{a n}{q}\left(m^{k}\right) \mid\right)+O\left(\frac{1}{L} \sum_{m \in B}\left|a_{m}\right|\right) .\right.
$$

Proof. This may be established in the same way as [6, identity (4)].

Lemma 2. Suppose that $\left\{a_{d}\right\}$ is a sequence of real numbers, all of the same sign and $a_{d} \ll N^{\eta}$. Then:

$$
\sum_{d<D} a_{d}\left|A_{d}\right|=2 \delta \sum_{d<D} a_{d}\left|B_{d}\right|+O\left(\delta \sum_{d<D}\left|\sum_{n \leq L} a_{d} \sum_{m d \in B} \mathrm{e}\left(\frac{a n}{q}(m d)^{k}\right)\right|\right)+O\left(\delta N^{1-2 \eta}\right)
$$

provided $D \ll N^{l-\varepsilon}$.

Proof. This follows from Lemma 1 by noting that:

$$
\sum_{d<D} a_{d}\left|A_{d}\right|=\sum_{m \in A}\left(\sum_{d<D, d \mid m} a_{d}\right)
$$

In bounding the error terms arising in the asymptotic formulae, we shall require bounds on certain exponential sums, which are given in [1]. The bounds given there remain valid for the increased value of $\rho$ we consider here.

Lemma 3. Let $Y \ll N^{\vartheta}$, where $\vartheta=(k-(2 k+1) \rho) /(2 k-2)$. Then

$$
\sum_{n \leq L} \sum_{y-Y} \max _{M}\left|\sum_{x \leq M, x \leq N / y} \mathrm{e}\left(\frac{a n}{q}(x y)^{k}\right)\right| \ll N^{1-4 \eta}
$$

Proof. This is [1, Lemma 10]. The removal of the condition of coprimality does not affect the result. 
Lemma 4. For $N^{2 \rho} \ll M \ll N^{1-\sigma}$, we have

$$
\sum_{n \leq L} \sum_{x \leq N / M}\left|\sum_{y \sim M} b_{y} \mathrm{e}\left(\frac{a n}{q}(x y)^{k}\right)\right| \ll N^{1-4 \eta}
$$

where $\left\{b_{y}\right\}$ is any sequence of complex numbers with $\left|b_{y}\right| \leq 1$.

Proof. This is [1, Lemma 11].

We now reach the stage where asymptotic formulae of the form (1) are to be established. Let $\theta$ be as in Lemma 3 above.

Lemma 5. Suppose that $k \geq 4$ and $M \ll N^{1 / 2+2 \rho}, b_{m} \ll N^{\eta}$. Then

$$
\sum_{m \sim M} b_{m} S\left(A_{m}, N^{\varepsilon}\right)=\sum_{m-M} b_{m} S\left(B_{m}, N^{\varepsilon}\right) 2 \delta(1+R)
$$

whenever the right hand side is $\gg \delta N^{1-\eta}$. Here $R=O(Q(s))+O\left(\exp \left(-(\log N)^{1 / 2}\right)\right)$ where $s=\log \left(N^{\vartheta} / M\right) / \log \left(N^{\varepsilon}\right)>(\vartheta-(0 \cdot 5+2 \rho)) / \varepsilon$ and $Q(s)<\exp (-s \log s+s \log \log 3 s+$ $O(s))$.

Remark. For $k=4$, from now on, we shall take $\rho(4)=1 / 21-\varepsilon_{2}$, where $\varepsilon_{2}$ is a small positive constant. Of course, $\varepsilon_{2}$ can be absorbed into $\varepsilon$ in the thoerem and so it would not affect the result.

Proof. Write $r\left(A_{m}, d\right)=(\varphi(q) / q)(2 \delta N / m d)-\left|A_{m d}\right|$. By the fundamental lemma of Rosser's sieve [10], we have:

$$
S\left(A_{m}, N^{\varepsilon}\right)=\frac{2 \delta N}{m} V_{1}\left(N^{\varepsilon}\right) \frac{\varphi(q)}{q}[1+O(Q(s))]+O\left(\sum_{d<N^{\theta} / M} a_{d} r\left(A_{m}, d\right)\right) .
$$

Also, by the Fundamental Lemma ([5, Theorem 2.5]),

$$
S\left(B_{m}, N^{\varepsilon}\right)=\frac{N}{m} V\left(N^{\varepsilon}\right)(1+O(Q(s))+O(\exp (-\sqrt{\log N}))
$$

It suffices therefore to show that

$$
\sum_{m \sim M}\left|b_{m} \sum_{d<N^{\theta} / M} a_{d} r\left(A_{m}, d\right)\right| \ll \delta N^{1-2 \eta}
$$

Note that for some $c_{m} \in \mathbb{C}$, we have

$$
\begin{aligned}
\sum_{m \sim M}\left|b_{m} \sum_{d<N^{\theta} / M} a_{d} r\left(A_{m}, d\right)\right|= & \sum_{m-M} c_{m} \sum_{d<N^{\theta} / M} a_{d}\left(\frac{2 \delta N}{m d}-\left|A_{m d}\right|\right) \\
= & \sum_{j} \lambda_{j}\left(2 \delta \frac{N}{j}-\left|A_{j}\right|\right) \\
& \ll \delta \sum_{n \leq L}\left|\sum_{j} \lambda_{j} \sum_{j \in \in B} \mathrm{e}\left(\frac{a n}{q}(j r)^{k}\right)\right|+O\left(\delta N^{1-2 \eta}\right)
\end{aligned}
$$


by Lemma 2 . The $\lambda_{j}$ are complex numbers with $\left|\lambda_{j}\right| \ll N^{\eta}$. The result now follows from Lemma 3. Then

LemMa 6. Suppose that $N^{2 \rho} \leq M \leq N^{1-\sigma}, a_{m}, b_{n}, c_{r} \ll N^{\eta}$ with $c_{r}=0$ whenever $r<N^{\varepsilon}$.

$$
\sum_{m r \sim M} a_{m} c_{r} \sum_{n \sim X} b_{n} S\left(A_{m n r}, r\right)=2 \delta \sum_{m r-M} a_{m} c_{r} \sum_{n \sim X} b_{n} S\left(B_{m n r}, r\right)(1+R)
$$

whenever the right hand side is $\gg \delta N^{1-\eta}$. Here $R=O(1 / \log N)$.

Proof. By Lemma 1, we have

$$
\begin{aligned}
& \sum_{m r \sim M} a_{m} c_{r} \sum_{n \sim X} b_{n} S\left(A_{m n r}, r\right)=\sum_{m r \sim M} a_{m} c_{r} \sum_{n \sim X} b_{n} \sum_{\substack{k m n r \in A, p \mid k=p \geq r}} 1= \\
& 2 \delta(1+R) \sum_{m r \sim M} a_{m} c_{r} \sum_{n \sim X} b_{n} S\left(B_{m n r}, r\right)+O\left(\delta \sum_{l \leq L} \mid \sum_{m r \sim M} a_{m} c_{r} \sum_{n \sim X} b_{n} \sum_{\substack{w n m r \in B \\
p \mid w \Rightarrow p \geq r}} \mathrm{e}\left(\frac{a j}{q}(w n m r)^{k}\right)\right.
\end{aligned}
$$

where $R=O(1 / \log N)$.

It remains to show that the exponential sum is $\ll N^{1-2 \eta}$. To achieve this, we have recourse to the following result $([3$, p. 165]):

$$
\begin{aligned}
& \int_{\Gamma} \mathrm{e}^{i t a} \frac{\sin b t}{\pi t} d t=\Delta(a, b)+O\left(\frac{1}{T(b-|a|)}\right) \text {, where } \Delta(a, b)=1 \text { if }|a| \leq b, \\
& \Delta(a, b)=0 \text { if }|a|>b .
\end{aligned}
$$

Here $\Gamma$ is $[-T, T]$.

The variable $w$ in the inner sum is a product of at most $s$ distinct primes where $s \ll 1 / \varepsilon$. Write

$$
\sum_{m r \sim M} a_{m} c_{r} \sum_{n \sim X} b_{n} \sum_{w m n r \in B, p \mid w=p \succeq r} \mathrm{e}\left(\frac{a j}{q}(w m n r)^{k}\right)=\sum_{1}+\sum_{2}+\ldots+\sum_{s}
$$

where, for $t=1,2, \ldots, s$,

$$
\sum_{t}=\sum_{m r \sim M} a_{m} c_{r} \sum_{n \sim X} b_{n} \sum_{\substack{p_{1} \ldots p_{1} n m r \in B \\ r<p_{1} \leq p_{2} \leq \ldots \leq p_{t}}} \mathrm{e}\left(\frac{a j}{q}\left(p_{1} \ldots p_{t} n m r\right)^{k}\right)
$$

To get rid of the inter-dependence of the variables, we apply (4) $t$ times, with $T$ being a suitable power of $N$. Each application incurs a factor of $O(\log N)$ due to the integration. For example, to get rid of the condition $p_{i} \leq p_{j}$, we let $a=p_{i}$ and $b=p_{j}+1 / 2$.

$$
\sum_{t} \ll_{\eta} N^{\eta}\left|\sum_{m r \sim M} a_{m} c_{r} \sum_{n \sim X} b_{n} \sum_{p_{1} \ldots p_{t} m n r \in B} \mathrm{e}\left(\frac{a j}{q}\left(p_{1} \ldots p_{t} n m r\right)^{k}\right) \lambda\left(p_{1}\right) \ldots \lambda\left(p_{t}\right)\right|
$$


where $\left|\lambda\left(p_{k}\right)\right| \leq 1$. Therefore:

$$
\begin{aligned}
\sum_{m r \sim M} a_{m} c_{r} \sum_{n \sim X} b_{n} \sum_{\substack{w m m r \in B \\
p \mid w \Rightarrow p>r}} \mathrm{e}\left(\frac{a j}{q}(w m n r)^{k}\right) \\
\qquad \ll_{\eta, \varepsilon} N^{\eta}\left|\sum_{m r \sim M} a_{m} c_{r} \sum_{n \sim X} b_{n} \sum_{w m n r \in B} u_{w} \mathrm{e}\left(\frac{a j}{q}(w m n r)^{k}\right)\right|
\end{aligned}
$$

where $\left|u_{w}\right| \leq 1$. The sum on the right hand side is

$$
\ll_{\eta, \varepsilon} N^{\eta}\left|\sum_{n \leq N / M} d_{n} \sum_{m \sim M, m n \in B} \alpha_{m} \mathrm{e}\left(\frac{a l}{q}(m n)^{k}\right)\right|
$$

for some $d_{n}$ and $\alpha_{m}$ whose magnitudes are $O_{\eta}\left(N^{\eta}\right)$ for each $n$ and $m$. We apply (4) again to eliminate the dependence between the variables $m$ and $n$, at the cost of a factor of $O(\log N)$. Bringing in the summation over $l$, the result now follows from Lemma 4 .

The following lemma allows us to control the error that accumulates as we iterate using the fundamental Buchstab identity, and which was not dealt with accurately in [6].

Lemma 7. Let $E$ be any subset of $B$. Let $t=[1 / \varepsilon]$, where $[x]$ denotes the integer part of $x$. Then

$$
\sum_{1 \leq h \leq t} \sum_{N^{\varepsilon} \leq p_{1} \leq \ldots \leq p_{h}} S\left(E_{p_{1} \ldots p_{h}}, N^{\varepsilon}\right) \leq 2^{t} S\left(E, N^{\varepsilon}\right)
$$

Proof. This is Lemma 10 in [2].

We are now in a position to prove our main versions of (1). To simplify the notation, we write $\xi=N^{2 p}$ and $\zeta=N^{1-\sigma}$.

Lemma 8. Suppose that $M \leq \zeta, a_{m} \ll N^{\eta}, a_{m} \geq 0$, and $a_{m}=0$ if $p|m \Rightarrow p<\zeta| \xi$. Then

$$
\sum_{m-M} a_{m} S\left(A_{m}, \zeta / \xi\right)=\sum_{m \sim M} a_{M} S\left(B_{m}, \zeta / \xi\right) 2 \delta(1+R)
$$

whenever the R.H.S. $\gg \delta N^{1-\eta}$. Here:

$$
R=O_{\varepsilon}\left(\frac{1}{\log N}\right)+O\left(2^{1 / \varepsilon} Q\left(\frac{\theta-0 \cdot 5+2 \rho}{\varepsilon}\right)\right) .
$$

Proof. If $M \geq N^{2 \rho}$, this follows immediately from Lemma 6, so we may assume $M<N^{2 \rho}$. Apply Buchstab's identity to obtain

$$
\sum_{m \sim M} a_{m} S\left(A_{m}, \zeta / \xi\right)=\sum_{m \sim M} a_{m} S\left(A_{m}, N^{\varepsilon}\right)-\sum_{m-M} a_{m} \sum_{N^{e} \leq p<\zeta / \xi} S\left(A_{m p}, p\right)
$$

We can deal with the first sum using Lemma 5. For the subsum in the second sum with $m p \geq \xi$, we could apply Lemma 6 . For the remaining parts, we apply Buchstab's identity:

$$
\sum_{m \sim M} a_{m} \sum_{\substack{N^{\varepsilon} \leq p<\xi / \xi \\ m p<\xi}} S\left(A_{m p}, p\right)=\sum_{m-M} a_{m} \sum_{\substack{N^{\varepsilon} \leq p<\xi / \xi \\ m p<\xi}}\left(S\left(A_{m p}, N^{\varepsilon}\right)-\sum_{N^{e} \leq r<p} S\left(A_{m p r}, r\right)\right) .
$$

The first term can again be handled by Lemma 5 . The parts in the second term with 
$m p r \geq \xi$ can be dealt with by Lemma 6. As before, we apply Buchstab's identity to the remaining parts.

Continuing in this way, we have the result above after $O(1 / \varepsilon)$ steps. The error can be at most

$$
\sum_{1 \leq h \leq t} \delta \sum_{m \sim M} a_{m} \sum_{N^{\varepsilon} \leq p_{1} \ldots<P_{h}} S\left(B_{m p_{1} \ldots p_{h}}, N^{\varepsilon}\right)\left(K_{1} Q(s)+K_{2}\left(\frac{1}{\log N}\right)\right)
$$

where $t=[1 / \varepsilon]$ and $s=(\theta-(0 \cdot 5+\rho)) / \varepsilon . K_{1}$ is an absolute constant while $K_{2}$ may depend on $\varepsilon$. By Lemma 7 , this is

$$
\begin{aligned}
& \ll \delta \sum_{m-M} a_{m} 2^{t} S\left(B_{m}, N^{\varepsilon}\right)\left(K_{1} Q(s)+K_{2}\left(\frac{1}{\log N}\right)\right) \\
& \ll \delta \sum_{m \sim M} a_{m} \frac{2^{t}}{\varepsilon} S\left(B_{m}, \zeta / \xi\right)\left(K_{1} Q(s)+K_{2}\left(\frac{1}{\log N}\right)\right) .
\end{aligned}
$$

Note that $2^{t} Q(s) / \varepsilon \rightarrow 0$ as $\varepsilon \rightarrow 0$. This establishes the result.

Similarly, we may prove the following. Then

Lemma 9. For $k \geq 4$, and $\zeta<M \leq N^{1 / 2}, 0 \leq a_{m} \leq N^{\eta}$, and $a_{m} \neq 0 \Rightarrow p \geq \xi$ for all $p \mid m$.

$$
\sum_{m \sim M} a_{m} S\left(A_{m}, \xi\right)=\sum_{m \sim M} a_{m} S\left(B_{m}, \xi\right) 2 \delta(1+R)
$$

whenever the R.H.S. is $\gg \delta N^{1-\eta}$. Here $\left.R=O\left(2^{1 / \varepsilon} \varepsilon^{-1} Q(1 / \varepsilon)\right)+O_{\varepsilon}(1 / \log N)\right)$.

Proof. This is similar to that of Lemma 8. The reason for the value $\xi$ is that it is the maximum value for which $M \xi \leq N^{\vartheta}$ with equality possible for $k=4$, a condition required for the application of Lemma 5.

4. Proof of Theorem for $k \geq 4$. We decompose $S\left(A, N^{1 / 2}\right)$ as follows:

$$
S\left(A, N^{1 / 2}\right)=S(A, \zeta / \xi)-\sum_{\zeta / \xi \leq p \leq \zeta} S\left(A_{p}, p\right)-\sum_{\zeta<p<N^{1 / 2}} S\left(A_{p}, \xi\right)+\sum_{\zeta<p<N^{1 / 2}} \sum_{\xi \leq r<p} S\left(A_{p r}, r\right) .
$$

Note that the condition $p r^{2}<N$ is implicit in the last sum. By Lemmas 8 and 9, we can give asymptotic formulae for the first three terms. In the double sum, we can give an asymptotic formula for the part with $\xi \leq r \leq \zeta$ by Lemma 6 . We discard the rest. This gives an overall lower bound for $S\left(A, N^{1 / 2}\right)$ :

$$
S\left(A, N^{1 / 2}\right) \geq 2 \delta \frac{N}{\log N}\left(1-\frac{\log N}{N} \sum_{\zeta<p<N^{1 / 2}} \sum_{\zeta<r<p} S\left(B_{p r}, r\right)\right) .
$$

We shall estimate the amount discarded using the prime number theorem and the asymptotic formula:

$$
S\left(B_{n}, z\right)=\frac{N}{n \log N} \omega\left(\frac{\log (N / n)}{\log z}\right)\left(1+O\left(\frac{1}{\log N}\right)\right) .
$$


Here, $\omega(u)$ is Buchstab's function which satisfies:

$$
\begin{array}{ll}
\omega(u)=1 / u & \text { for } 1 \leq u \leq 2, \\
\omega(u)=(1+\log (u-1) / u & \text { for } 2 \leq u \leq 3, \\
\omega(u)<0.5644 & \text { for } u>3 .
\end{array}
$$

We can replace the double sums by the double integral (with a negligible error)

$$
\iint_{\Delta} \omega\left(\frac{1-\alpha-\beta}{\beta}\right) \frac{d \alpha d \beta}{\alpha \beta^{2}}
$$

where $\Delta:=\{(\alpha \beta): 1-\sigma \leq \alpha \leq 1 / 2,1-\sigma \leq \beta \leq \min (\alpha,(1-\alpha) / 2)\}$.

By a computer calculation, one can show that the amount discarded is $<1$. Hence the result is proved. The limit of the value for $\rho(4)$ is set by the condition that $1 / 2+2 \rho \leq \vartheta$, which is required in establishing Lemma 5 and Lemma 9. The limit for the other values is set by the fact that we cannot decompose the double sums into manageable parts with discardable positive sums.

5. The case of $k=3$. For the above to work, we require at least $\vartheta \geq 1 / 2+2 \rho$. For $k=3$, this leads to $\rho \leq 1 / 15$. Thus, for an improvement, a trick is required to circumvent this difficulty. With the introduction of this new idea, the restriction can now be relaxed to $\vartheta \geq \rho+1 / 2$. This gives $\rho \leq 1 / 11$. The following is the consequence of Lemmas 5 and 6 with $k=3$. To simplify the notation, write $\tau=N^{1 / 2-\rho}, v=N^{1 / 2+\rho}$.

LEMma 10. For $M \leq \tau, a_{m} \ll N^{\eta}$, we have

$$
\sum_{m \sim M} a_{m} S\left(A_{m}, \zeta / \xi\right)=\sum_{m \sim M} a_{m} S\left(B_{m}, \zeta / \xi\right) 2 \delta(1+R)
$$

where $R$ is as in Lemma 8.

It is clear that simply using the lemmas obtained so far, we cannot give an asymptotic formula for the sum $\sum S\left(A_{p}, N^{c}\right)$ for the range $\tau<p<N^{1 / 2}$, for some suitable value $c$ as before. We decompose the sum $S\left(A, N^{1 / 2}\right)$ as follows:

$$
\begin{aligned}
S\left(A, N^{1 / 2}\right)= & S(A, \zeta / \xi)-\sum_{\zeta / \xi \leq p \leq \zeta} S\left(A_{p}, p\right)-\sum_{\zeta<p \leq \tau} S\left(A_{p}, p\right)-\sum_{\tau<p<N^{1 / 2}} S\left(A_{p}, p\right) \\
= & S(A, \zeta / \xi)-\sum_{\zeta / \xi \leq p \leq \xi} S\left(A_{p}, \zeta / \xi\right)-\sum_{\xi \leq p \leq \zeta} S\left(A_{p}, p\right)-\sum_{\zeta<p \leq \tau} S\left(A_{p}, \zeta / \xi\right) \\
& -\sum_{\tau<p<N^{1 / 2}} S\left(A_{p}, p\right)+\sum_{\zeta / \xi \leq p<\xi} \sum_{\zeta / \xi \leq q<p} S\left(A_{p q}, q\right)+\sum_{\zeta<p \leq \tau} \sum_{\zeta / \xi \leq q<p} S\left(A_{p q}, q\right) \\
= & S_{1}-S_{2}-S_{3}-S_{4}-S_{5}+S_{6}+S_{7}
\end{aligned}
$$

say. We may give asymptotic formulae for $S_{1}, S_{2}, S_{3}, S_{4}$ by Lemmas 6 and 10 . We may give asymptotic formulae for the subsums of $S_{6}$ where $p q<\zeta$. We may also give an asymptotic formula for the subsums of $S_{7}$ with $\zeta / \xi \leq q<\zeta^{1 / 2}$ using similar methods in establishing Lemma 8 : here, if $\zeta^{1 / 2} \geq p_{1} \geq p_{2} \geq p_{3} \geq \ldots \geq p_{h}, p_{1} \ldots p_{h-1} \leq \xi, p_{1} \ldots p_{h} \geq \xi$, then 
$p p_{1} \ldots p_{h-1} \leq N^{1 / 2+\rho}$, also $p_{1} \ldots p_{h} \leq N^{1-8 \rho} \leq \zeta$. We now deal with $S_{5}$. By Buchstab's identity, we have

$$
S_{5}=\sum_{\tau<p<N^{12}} S\left(A_{p}, N^{\varepsilon}\right)-\sum_{\tau<p<N^{12}} \sum_{N^{\varepsilon} \leq q<p} S\left(A_{p q}, q\right),
$$

The second sum can be written as a sum of four sums $T_{1}, T_{2}, T_{3}, T_{4}$ where $T_{1}=\sum S\left(A_{p q}, q\right)$ with $\tau<p<N^{1 / 2}$ and $N^{\varepsilon} \leq q<\zeta / \xi, T_{2}=\sum S\left(A_{p q}, q\right)$ with $\tau<p<N^{1 / 2}$ and $\zeta / \xi<q<\xi$, $T_{3}=\sum S\left(A_{p q}, q\right)$ with $\tau<p<N^{1 / 2}$ and $\xi \leq q<\zeta$, and $T_{4}=\sum S\left(A_{p q}, q\right)$ with $\tau<p<N^{1 / 2}$ and $q>\zeta$. We shall discard $T_{2}$ and $T_{4} . T_{3}$ can be dealt with using Lemma 6. For $T_{1}$, since $p q<v$, we may apply Buchstab's identity:

$$
T_{1}=\sum S\left(A_{p q}, N^{\varepsilon}\right)-\sum_{N^{\varepsilon} \leq t<q} S\left(A_{p q t}, t\right)
$$

By Lemma 5, we may give an asymptotic formula for the first sum. We can also give an asymptotic formula for subsums in the second sum with $q t \geq \xi$ (since it is necessary that $q t<\zeta)$. For the remainder, we split the sum again:

$$
\sum_{q t<N^{2 p}} S\left(A_{p q t}, t\right)=\sum_{p q t \leq v} S\left(A_{p q t}, t\right)+\sum_{p q r>v} S\left(A_{p q t}, t\right),
$$

We first consider the first term. We apply Buchstab's identity:

$$
\sum_{p q t \leq v} S\left(A_{p q t}, t\right)=\sum S\left(A_{p q t}, N^{\varepsilon}\right)-\sum_{N^{\varepsilon} \leq h<t} S\left(A_{p q t h}, h\right) .
$$

It is necessary that $q t h \leq \zeta$, so that subsums in the second term are manageable if $q t h \geq \xi$. Split the remaining subsum according to whether $p q t h>v$ or $p q t h \leq v$. Thus, it suffices to consider sums of the form

$$
\sum_{p q>v} S\left(A_{p q}, q\right), \quad \sum_{p q r_{2}>v} \sum_{N^{e} \leq q<\xi / \xi} \sum_{N^{\varepsilon} \leq r_{2}<q, q r_{2}<\xi} S\left(A_{p q r_{2}}, r_{2}\right), \ldots \text { etc. }
$$

We use $\Sigma^{*}$ to denote the sum over the ranges: $\tau<p \leq N^{1 / 2}, N^{\varepsilon} \leq r_{m}<r_{m-1}<\ldots<r_{1} \leq$ $\zeta / \xi, r_{1} r_{2} \ldots r_{m}<\xi, p r_{1} r_{2} \ldots r_{m}>v, p r_{1} r_{2} \ldots r_{m-1} \leq v$. Thus, the general form of the sum we have to consider is

$$
\sum^{*} S\left(A_{p r_{1} \ldots r_{m}}, r_{m}\right)
$$

The "different technique" mentioned in the introduction involves writing

$$
\sum_{p r_{1} \ldots r_{m}>v}^{*} S\left(A_{p r_{1} \ldots r_{m}}, r_{m}\right)=\sum_{s \mid x \rightarrow s>r_{m}}^{*} S\left(A_{x r_{1} \ldots r_{m}},\left(\frac{N}{x r_{1} \ldots r_{m}}\right)^{1 / 2}\right) .
$$

We remark that $x \leq \tau$ and $N /\left(x r_{1} \ldots r_{m}\right)$ has the size of $p$. Thus, we may apply Buchstab's identity:

$$
\sum_{s \mid x \Rightarrow s>r_{m}}^{*} S\left(A_{x r_{1} \ldots r_{m}},\left(\frac{N}{x r_{1} \ldots r_{m}}\right)^{1 / 2}\right)=\sum_{x}^{*} S\left(A_{x r_{1} \ldots r_{m}}, N^{\varepsilon}\right)-\sum_{N^{\varepsilon} \leq r<\left(N / r_{1} \ldots r_{m} x\right)^{12}} S\left(A_{x r_{1} \ldots r_{m} r} r\right) .
$$

By Lemma 5, the first term can be dealt with. By Lemma 6, the subsums in the second sum are manageable if $r \geq \xi$ (since it is necessary that $r<\zeta$ ). For the remainder, we observe that when $\zeta / \xi \leq r \leq \xi, r_{1} \ldots r_{m} \geq \zeta / \xi$. $N^{p} \geq \xi$, as it is necessary that $r_{1} \ldots r_{m} \geq N^{\rho}$. 
Thus, by Lemma, 6 we may give an asymptotic formula for the subsum of the second sum with $\zeta / \xi \leq r \leq \xi$.

For the part with $r<\zeta / \xi$, we have an iterative process: the sum with $r_{1} \ldots r_{m} r \geq \xi$ is manageable by Lemma 6 . If $r_{1} \ldots r_{m} r<\xi$, we iterate again and so we eventually obtain the desired result after $O(1 / \varepsilon)$ operations. Thus, we may give an asymptotic formula for $T_{1}$ with an error of suitable size, similar to that in Lemma 8 or 9.

We discard $T_{2}, T_{4}$ and the subsums of $S_{5}$ and $S_{7}$ for which we fail to give asymptotic formulae. We approximate those sums by integrals as before. This produces a lower bound of

$$
\frac{\delta N}{\log N}\left[1-\left(\iint_{D_{1}}+\iint_{D_{2}}+\iint_{D_{3}}\right) \omega\left(\frac{1-\alpha-\beta}{\beta}\right) \frac{d \alpha d \beta}{\alpha \beta^{2}}\right]
$$

where

$$
\begin{aligned}
D_{1} & =\{(\alpha, \beta): \alpha+\beta \geq 1-8 \rho, 1-10 \rho<\beta<\alpha<2 \rho\}, \\
D_{2} & =\{(\alpha, \beta): \beta \leq \min (\alpha, 1-2 \alpha), \beta>1-8 \rho\}, \\
D_{3} & =\{(\alpha, \beta): 1-8 \rho<\alpha \leq 1 / 2-\rho,(1-8 \rho) / 2 \leq \beta \leq 2 \rho\} \\
& \cup\{(\alpha, \beta): 1 / 2-\rho<\alpha \leq 1 / 2,1-10 \rho \leq \beta \leq 2 \rho\} .
\end{aligned}
$$

The function $\omega$ is Buchstab's function as given in section 4. The region $D_{1}$ corresponds to the subsum of $S_{6}$ to be discarded and $D_{2}$ corresponds to $T_{4}$ and the subsum of $S_{7}$ with $q>\zeta$, while $D_{3}$ corresponds to the subsum of $S_{7}$ with $\zeta / \xi \leq q<\xi$ and $T_{2}$. We evaluate these integrals by machine calculations. With $\rho=5 / 56$, we have

$$
\begin{aligned}
& \iint_{D_{1}} \omega\left(\frac{1-\alpha-\beta}{\beta}\right) \frac{d \alpha d \beta}{\alpha \beta^{2}}<0.22, \\
& \iint_{D_{2}} \omega\left(\frac{1-\alpha-\beta}{\beta}\right) \frac{d \alpha d \beta}{\alpha \beta^{2}}<0.10, \\
& \iint_{D_{3}} \omega\left(\frac{1-\alpha-\beta}{\beta}\right) \frac{d \alpha d \beta}{\alpha \beta^{2}}<0.65 .
\end{aligned}
$$

Thus, we do have a positive lower bound, so our result is proved. The value of $\rho$ may be improved slightly, but it is noted that, with $\rho=6 / 67$, the amount discarded would be greater than 1.

ACKNowledGement. I would like to thank G. Harman for his encouragement and valuable suggestions, and EPSRC for financial support. I would also like to thank the referee for useful comments.

\section{REFERENCES}

1. R. C. Baker and G. Harman, On the distribution of $\alpha p^{k}$ modulo one, Mathematika 38 (1991), 170-184. 
2. R. C. Baker, G. Harman and J. Rivat, Primes of the form $\left[n^{c}\right], J$. Number Theory 50 (1995), 261-277.

3. H. Davenport, Multiplicative number theory (second edition, revised by H. L. Montgomery), (Springer, New York-Heidelberg-Berlin, 1980). $252-269$

4. A. Ghosh, The distribution of $\alpha p^{2}$ modulo one, Proc. London Math. Soc. (3), 42 (1981),

5. H. Halberstam and H.-E. Richert, Sieve methods (Academic Press, 1974). 9-18.

6. G. Harman, On the distribution of $\alpha p$ modulo one, J. London Math. Soc. (2) 27 (1983),

7. G. Harman, Trigonometric sums over primes I, Mathematika, 28 (1981), 249-254.

8. G. Harman, Trigonometric sums over primes II, Glasgow Math. J. 24 (1983), 23-37.

9. G. Harman, On the distribution of $\alpha p$ modulo one II, Proc. London Math. Soc. (3) 72 (1996), 241-260.

10. H. Iwaniec, Rosser's sieve, Acta Arithmetica 36 (1980), 171-202.

11. C.-H. Jia, On the distribution of $\alpha p$ modulo one, J. Number Theory 45 (1993), 241-253.

12. R. C. Vaughan, On the distribution of $\alpha p$ modulo one, Mathematika 24 (1977), 135-141.

13. I. M. Vinogradov, The method of trigonometric sums in the theory of numbers (translated, revised and annotated by A. Davenport and K. F. Roth) (Interscience, New York, 1954).

14. I. M. Vinogradov, A general distribution law for the fractional parts of a polynomial with variables running over primes, C.R. Doklady, Acad.-Sci. URSS(NS) 51 (1946), 491-492.

School of Mathematics.

University of Wales, College of Cardiff

SENGHENYDD ROAD,

CARDIFF CF2 4AG. 